\title{
Variaciones Anatómicas de la Arteria Hepática: Patrones Vasculares Observados en 140 Hígados de Donante Cadavérico
}

\author{
Anatomical Variations of the Hepatic Artery: \\ Vascular Patterns Observed in 140 Cadaveric Donor Livers \\ Tomás Cifone'; Santiago Lihué Lassalle'; Victoria Cristina Serrano Bernárdez; \\ Agostina Roggero' ${ }^{1}$ Nicolás Benitez ${ }^{1}$; Verónica Garay ${ }^{1,2}$ \& Mariana Bendersky ${ }^{1}$
}

\begin{abstract}
CIFONE, T.; LASSALLE, S. L.; SERRANO BERNÁRDEZ, V. C.; ROGGERO, A. ; BENITEZ, N.; GARAY, V. \& BENDERSKY, M. Variaciones anatómicas de la arteria hepática: Patrones vasculares observados en 140 hígados de donante cadavérico. Int. J. Morphol., 39(5):1391-1394, 2021.

RESUMEN: El objetivo de este trabajo es analizar las variantes de la arteria hepática observadas en hígados de donantes cadavéricos empleados para trasplante ortotópico de hígado en nuestra población. Se efectuó un estudio retrospectivo de una cohorte de 140 trasplantes entre junio del año 2011 y enero del año 2021. La anatomía vascular arterial de los injertos hepáticos fue clasificada de acuerdo a la descripción de Hiatt. Variante clásica de la arteria hepática - Tipo I: 62 casos $(65,7 \%)$. Variante no clásica de la arteria hepática: 48 casos (34,3\%), correspondientes a: Tipo II: 12 casos (8,6\%), Tipo III: 18 casos (12,9\%), Tipo IV: 7 casos (5 \%), Tipo V: 10 casos (7,1 \%). No se encontró ningún caso de variante Tipo VI. Se halló 1 caso $(0,7 \%)$ no descrito en esta clasificación correspondiente a una arteria hepática izquierda accesoria que se originaba de la aorta. El conocimiento preciso de las variaciones más prevalentes, y también de las menos frecuentes, es fundamental para los procedimientos quirúrgicos que se realizan en el abdomen superior.
\end{abstract}

PALABRAS CLAVE: Arteria hepática; Trasplante hepático; Anatomía; Circulación hepática; Cirugía general.

INTRODUCCIÓN

Desde las primeras descripciones precisas en el siglo XVIII realizadas por los padres de la angiología moderna J.B. Winslow y A. Haller, es bien conocido que la arteria hepática a menudo presenta patrones vasculares que difieren al de la descripción clásica (Haller, 1756).

En el año 1966, Michels (1966) se convierte en uno de los primeros autores en establecer una clasificación internacional a través de la disección de 200 cadáveres, englobando las distintas variaciones de la arteria hepática en 10 patrones. A partir de esta publicación varios estudios, no sólo han reportado múltiples variaciones tanto frecuentes como raras de la arteria hepática, sino que también han intentado determinar diferentes clasificaciones. Estos estudios se han basado en disecciones cadavéricas, imágenes angiográficas y trasplantes de hígado, entre otras cirugías (Suzuki et al., 1971; Rygaard et al., 1986). En el año 1994, Hiatt et al. (1994) a través del análisis de 1000 trasplantes de hígado simplifican la clasificación de Michels reduciéndo- la a 6 patrones. A esta última se la conoce como clasificación de Hiatt o clasificación de Michels modificada (Fig. 1).

La anatomía de la arteria hepática tiene gran importancia en la cirugía general y en la cirugía hepática, especialmente en el trasplante de hígado, así como en múltiples procedimientos de intervencionismo radiológico como la quimioembolización de tumores hepáticos (Abdullah et al., 2006).

La cirugía de ablación hepática en donante cadavérico y los procedimientos de la cirugía de banco (realizados antes de la implantación del órgano) ofrecen una oportunidad única para la identificación precisa de los diferentes patrones de la arteria hepática (López-Andújar et al., 2007).

El objetivo de este trabajo fue analizar las variantes de la arteria hepática observadas en 140 hígados de donantes cadavéricos empleados para trasplante ortotópico de hígado en nuestra población.

\footnotetext{
${ }^{1}$ Laboratorio de Anatomía Viviente(UBACyT), III Cátedra de Anatomía. Facultad de Medicina, Universidad de Buenos Aires (UBA), Argentina.

${ }^{2}$ Programa de Trasplante Hepático, Hospital General de Agudos "Dr. Cosme Argerich", Ciudad Autónoma de Buenos Aires, Argentina.
} 

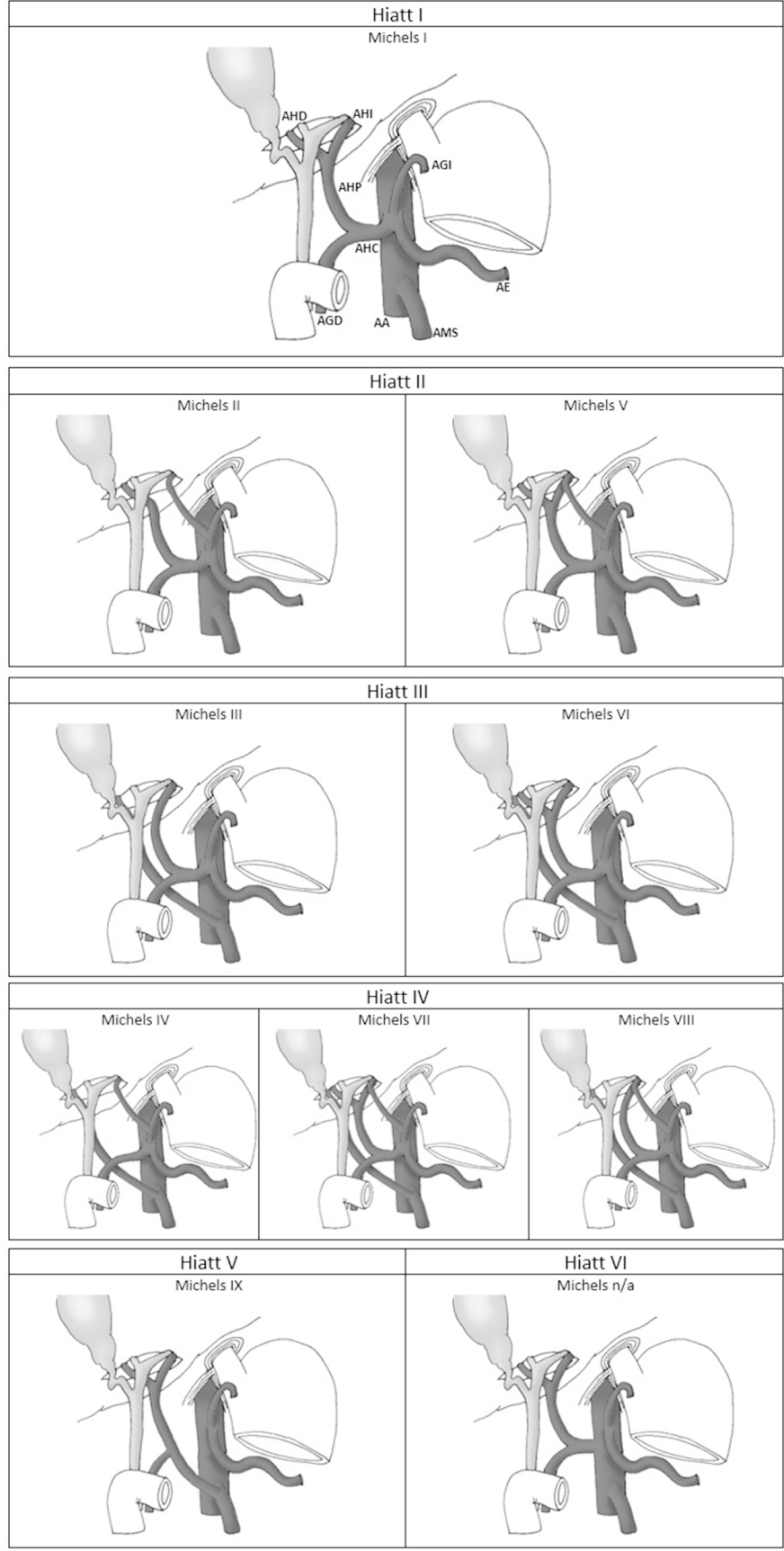

Fig. 1. Representación de la clasificación de Hiatt y su correlación con la clasificación de Michels. AA. Parte abdominal de la aorta; AGI. Arteria gástrica izquierda; AE. Arteria esplénica; AHC. Arteria hepática común; AGD. Arteria gastroduodenal; AHP. Arteria hepática propia; AHD. Arteria hepática derecha; AHI. Arteria hepática izquierda; AMS. Arteria mesentérica superior.

\section{MATERIAL Y MÉTODO}

Se realizó un análisis retrospectivo de un total de 140 casos de trasplante ortotópico de hígado llevados a cabo, entre junio del año 2011 y enero del año 2021, en el Servicio de Trasplante Hepático del Hospital General de Agudos "Dr. Cosme Argerich" de la Ciudad de Buenos Aires. Se analizaron los registros operatorios de los 140 injertos de donante cadavérico, cuya anatomía arterial hepática se encontraba descrita tanto en los partes quirúrgicos de ablación como en los de implante del órgano.

Se adoptó como esquema arterial clásico: una arteria hepática común, rama del tronco celíaco, que alcanza al hígado a través de la arteria hepática propia bifurcándose, esta última, en arterias hepáticas derecha e izquierda; en ausencia de arterias hepáticas accesorias.

Los patrones de irrigación hepática se documentaron según la clasificación de Hiatt.

\section{RESULTADOS}

La irrigación hepática clásica (Tipo I) se encontró en 92 hígados $(65,7 \%)$. Los demás, es decir, 48 injertos $(34,3 \%)$ presentaron arterias hepáticas aberrantes.

De acuerdo a la clasificación de Hiatt, los patrones no clásicos observados con mayor frecuencia fueron: una arteria hepática derecha accesoria o reemplazante que se originó de la arteria mesentérica superior (Tipo III) en 18 casos (12,9\%); una arteria hepática izquierda accesoria o reemplazante que se originó de la arteria gástrica izquierda (Tipo II) en 12 casos $(8,6 \%)$; la arteria hepática común que se originó de la artería mesentérica superior (Tipo V) en 10 casos $(7,1 \%)$; una arteria hepática izquierda y una arteria hepática derecha accesorias o reemplazantes que se originaron de las arterias gástrica izquierda y mesentérica superior respectivamente (Tipo IV) en 7 casos (5\%). No se encontró ningún caso al descrito como Tipo VI, en el que la arteria hepática común se origina directamente de la aorta abdominal.

Además de los patrones arteriales mencionados anteriormente, que en general representan los patrones más frecuentemente reportados en la literatura, se observó un caso de una arteria hepática izquierda accesoria originándose directamente de la de la aorta abdominal (0,7 \%). Fig. 2. 


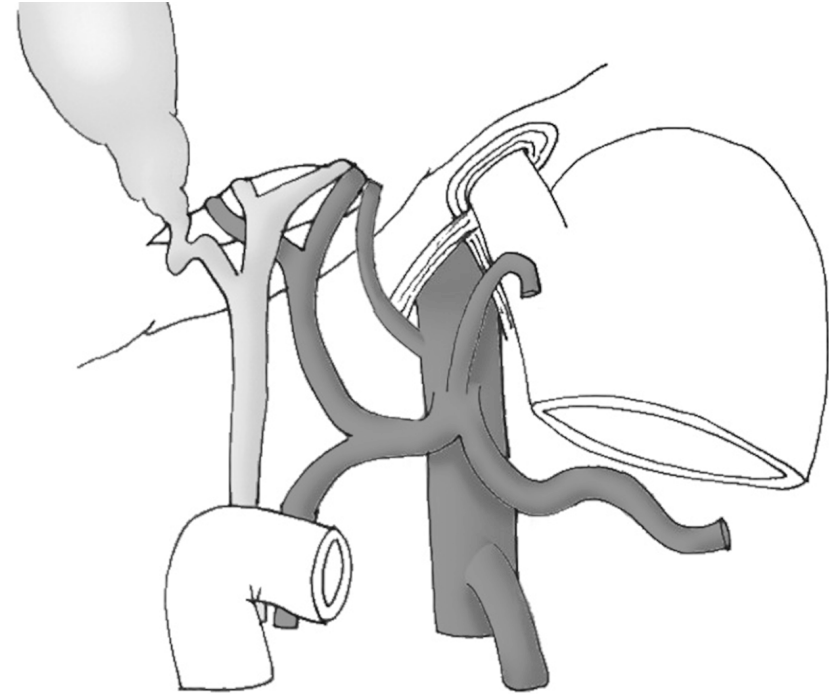

Fig. 2. Arteria hepática accesoria originándose de la parte abdominal de la aorta.

\section{DISCUSIÓN}

Si hay algo que caracteriza al sistema arterial del hígado es la elevada frecuencia de variaciones en su anatomía. En estos patrones aberrantes, los distintos segmentos hepáticos reciben el flujo arterial a través de ramas que se originan de las arterias mesentérica superior, gástrica izquierda $\mathrm{y}$, menos frecuentemente, de otros troncos arteriales (Imam et al., 2021).

Muchos de los órganos del abdomen superior, incluyendo el estómago, páncreas, bazo y duodeno, sufren un daño isquémico menor por la lesión de una rama arterial principal (Suzuki et al.). Dado que las ramas de la arteria hepática que irrigan el hígado son consideradas arterias terminales, en el contexto de un trasplante hepático todos los vasos, y especialmente los aberrantes del tipo reemplazantes, deben ser preservados y revascularizados para evitar complicaciones isquémicas del parénquima hepático y de las vías biliares (Merion et al., 1989). Es por ello que el trasplante hepático representa una oportunidad única a la hora de analizar las variaciones arteriales.

Según la literatura, las variaciones del esquema clásico ocurren aproximadamente entre el 20 y el $45 \%$ de los casos. Existen fundamentalmente tres formas de estudiar estas variables, que son: la disección cadavérica, los métodos de diagnóstico por imágenes y la cirugía, especialmente el trasplante hepático. Michels analizó 200 disecciones cadavéricas, encontrando un $45 \%$ de variaciones de la anatomía arterial hepática. Ottone et al. (2006), a través de 64 cadáveres disecados, identificaron un $26,6 \%$ de arterias hepáticas aberrantes. Sebben et al. (2013) encontraron un $40 \%$ de variaciones en 45 disecciones. Koops et al. (2004) identificaron un 20,9\% de variaciones de la arteria hepática a través de 604 angiografías selectivas del tronco celíaco y la arteria mesentérica superior. Kobayashi et al. (2014) analizaron 1200 casos de imágenes angiográficas, encontrando un $22,8 \%$ de variaciones. En cuanto a la prevalencia en el contexto de trasplante de hígado, Hiatt et al. identificaron un 24,3 $\%$ de variaciones del patrón clásico en una serie de 1000 trasplantes. López-Andújar et al. a través de una muestra de 1081 trasplantes hepáticos, encontraron un 29,6\% de variaciones. En este estudio, mediante el análisis de 140 casos de trasplante hepático, se encontró un 34,3\% de variaciones de la arteria hepática. Si promediamos los resultados de estos estudios la prevalencia de variaciones del sistema arterial hepático es de un $30 \%$.

De acuerdo a la clasificación de Hiatt, las variaciones más frecuentes identificadas en esta investigación coinciden con algunos autores de la literatura, así como también encontramos diferencias con otros estudios (Tabla I).

Del análisis realizado se puede determinar que existen dos sitios de origen que son los más frecuentes dentro de estas variaciones. El primero es la arteria mesentérica superior, la cual da origen a una arteria hepática derecha aberrante. Esta última se dirige hacia el hilio hepático tomando, en primer lugar, un trayecto retroduodenal para luego ubicarse en el ligamento hepatoduodenal del omento menor. De esta

Tabla I. Comparación con otros estudios de las variaciones de la arteria hepática de acuerdo a la clasificación de Hiatt.

\begin{tabular}{|c|c|c|c|c|c|c|}
\hline Tipo & $\begin{array}{c}\text { Este estudio } \\
\text { (n: 140) } \\
\text { Trasplante }\end{array}$ & $\begin{array}{c}\text { Michels, } 1966 \\
\text { (n: 200) } \\
\text { Disección }\end{array}$ & $\begin{array}{l}\text { Hiatt et al., } 1994 \\
\text { (n: 1000) } \\
\text { Trasplante }\end{array}$ & $\begin{array}{c}\text { Abdullah et al., } \\
2006 \\
\text { (n: 932) } \\
\text { Trasplante }\end{array}$ & $\begin{array}{l}\text { López-Andújar } \\
\text { et al., 2007 } \\
\text { (n: 1081) } \\
\text { Trasplante }\end{array}$ & $\begin{array}{c}\text { Vasconcelos-Filho } \text { et } \\
\text { al., 2020 } \\
\text { (n: 340) } \\
\text { Trasplante }\end{array}$ \\
\hline I (\%) & 65,7 & 55 & 75,7 & 68,1 & 70,4 & 66,2 \\
\hline II (\%) & 8,6 & 18 & 9,7 & 8,1 & 13,6 & 13,5 \\
\hline III (\%) & 12,9 & 18 & 10,6 & 10,2 & 8,4 & 12,9 \\
\hline IV (\%) & 5 & 4 & 2,3 & 6,4 & 4 & 2,4 \\
\hline $\mathrm{V}(\%)$ & 7,1 & 4,5 & 1,5 & 1,6 & 2,5 & 2,4 \\
\hline Otros $(\%)$ & 0,7 & 0,5 & 0,2 & 5,6 & 1 & 2,6 \\
\hline
\end{tabular}


manera, forma parte del pedículo hepático en un plano más profundo que la arteria hepática propia y la vía biliar, siendo lateral a la vena porta. Es de suma importancia tener en cuenta esta disposición a la hora de la cirugía hepatobiliopancreática. El segundo sitio de origen es la arteria gástrica izquierda, la cual da origen a una arteria hepática izquierda aberrante que discurre por el sector posterosuperior del ligamento hepatogástrico del omento menor. Esta segunda variación cobra especial relevancia en la cirugía esofagogástrica además del trasplante hepático.

Más allá de los patrones más frecuentemente descritos en la literatura, identificamos una arteria hepática izquierda accesoria originándose directamente de la aorta abdominal y con un recorrido muy similar al de la arteria hepática izquierda aberrante que se origina de la arteria gástrica izquierda (Fig. 2). Este patrón ya ha sido descrito previamente por otros autores (Soin et al., 1996; Gruttadauria et al., 2001) con una prevalencia muy similar a la encontrada en este estudio.

En conclusión, las variaciones del patrón clásico de la arteria hepática se pueden encontrar en aproximadamente un tercio de los casos. El conocimiento preciso de las variaciones más prevalentes y también de las menos frecuentes es fundamental en las cirugías hepatobiliopancreáticas a fin de evitar lesiones y complicaciones intra y postoperatorias. Esta información se vuelve crítica en el trasplante hepático ya que la revascularización arterial del implante hepático es un factor relacionado en forma directa con la supervivencia tanto del receptor como del injerto.

CIFONE, T.; LASSALLE, S. L.; SERRANO BERNÁRDEZ, V. C.; ROGGERO, A. ; BENITEZ, N.; GARAY, V. \& BENDERSKY, M. Anatomical variations of the hepatic artery: Vascular patterns observed in 140 cadaveric donor livers. Int. J. Morphol., 39(5):13911394, 2021.

SUMMARY: The purpose of this article is to analyze the hepatic artery variations observed from the use of cadaveric donor livers for orthotopic transplantation among our population. A retrospective study of a liver transplant cohort including 140 donor livers was conducted between June 2011 and January 2021. The vascular arterial anatomy of the transplanted livers was classified according to Hiatt's classification system. Classic hepatic artery variant: Type I: 62 cases $(65.7 \%)$. Non-classic hepatic artery variants: 48 cases $(34.3 \%)$, corresponding to: Type II: 12 cases (8.6\%), Type III: 18 cases $(12.9 \%)$, Type IV: 7 cases (5\%), Type V: 10 cases $(7.1 \%)$. No case of Type VI variant was identified. One case $(0.7 \%)$ not included in Hiatt's classification was found, corresponding to an accessory left hepatic artery originating from the aorta. Precise knowledge regarding the most prevalent variations, as well as those that are the least common, is fundamental to upper abdominal surgical procedures.

KEY WORDS: Hepatic artery; Liver transplantation; Anatomy; Liver circulation; General surgery.

\section{REFERENCIAS BIBLIOGRÁFICAS}

Abdullah, S. S.; Mabrut, J. Y.; Garbit, V.; De La Roche, E.; Olagne, E.; Rode, A.; Morin, A.; Berthezene, Y.; Baulieux, J. \& Ducerf, C. Anatomical variations of the hepatic artery: study of 932 cases in liver transplantation. Surg. Radiol. Anat., 28(5):468-73, 2006.

Gruttadauria, S.; Foglieni, C. S.; Doria, C.; Luca, A.; Lauro, A. \& Marino, I. R. The hepatic artery in liver transplantation and surgery: vascular anomalies in 701 cases. Clin. Transplant., 15(5):359-63, 2001.

Haller, A. Icones Anatomicae in Quibus Aliquae Partes Corporis Humani Delineatae Proponuntur et Arteriarum Potissimum Historia Continetur. Gottingen, Vandenhoeck \& Ruprecht, 1756.

Hiatt, J. R.; Gabbay, J. \& Busuttil, R. W. Surgical anatomy of the hepatic arteries in 1000 cases. Ann. Surg., 220(1):50-2, 1994.

Imam, A.; Karatas, C.; Mecit, N.; K, A.; Yildirimoglu, T.; Kalayoglu, M. \& Kanmaz, T. Anatomical variations of the hepatic artery: a closer view of rare unclassified variants. Folia Morphol. (Warsz), 2021. DOI: https:// www.doi.org/10.5603/FM.a2021.0024

Kobayashi, S.; Otsubo, T.; Koizumi, S.; Ariizumi, S.; Katagiri, S.; Watanabe, T.; Nakano, H. \& Yamamoto, M. Anatomic variations of hepatic artery and new clinical classification based on abdominal angiographic images of 1200 cases. Hepatogastroenterology, 61(136):2345-8, 2014.

Koops, A.; Wojciechowski, B.; Broering, D. C.; Adam, G. \& Krupski-Berdien, G. Anatomic variations of the hepatic arteries in 604 selective celiac and superior mesenteric angiographies. Surg. Radiol. Anat., 26(3):239-44, 2004.

López-Andújar, R.; Moya, A.; Montalvá, E.; Berenguer, M.; De Juan, M.; San Juan, F.; Pareja, E.; Vila, J. J.; Orbis, F.; Prieto, M.; et al. Lessons learned from anatomic variants of the hepatic artery in 1,081 transplanted livers. Liver Transpl., 13(10):1401-4, 2007.

Merion, R. M.; Burtch, G. D.; Ham, J. M.; Turcotte, J. G. \& Campbell, D. A. The hepatic artery in liver transplantation. Transplantation, 48(3):438-43, 1989.

Michels, N. A. Newer anatomy of the liver and its variant blood supply and collateral circulation. Am. J. Surg., 112(3):337-47, 1966.

Ottone, N. E.; Arrotea Molina, A.; Domínguez, M. L.; Lo Tártaro, M.; García de Quiros, N.; Medan, C.; Blasi, E. \& Bertone, V. H. Arterias hepáticas aberrantes: estudio en 64 cadáveres disecados. Int. J. Morphol., 24(4):5815, 2006.

Rygaard, H.; Forrest, M.; Mygind, T. \& Baden, H. Anatomic variants of the hepatic arteries. Acta Radiol. Diagn., 27(4):425-7, 1986.

Sebben, G. A.; Rocha, S. L.; Sebben, M. A.; Parussolo Filho, P. R. \& Goncalves, B. H. Variations of hepatic artery: anatomical study on cadavers. Rev. Col. Bras. Cir., 40(3):221-6, 2013.

Soin, A. S.; Friend, P. J.; Rasmussen, A.; Saxena, R.; Tokat, Y.; Alexander, G. J.; Jamieson, N. V. \& Calne, R. Y. Donor arterial variations in liver transplantation: management and outcome of 527 consecutive grafts. $\mathrm{Br}$. J. Surg., 83(5):637-41, 1996.

Suzuki, T.; Nakayasu, A.; Kawabe, K.; Takeda, H. \& Honjo, I. Surgical significance of anatomic variations of the hepatic artery. Am. J. Surg., 122(4):505-12, 1971.

\section{Dirección para correspondencia:}

Tomás Cifone

Laboratorio de Anatomía Viviente (UBACyT)

III Cátedra de Anatomía. Facultad de Medicina

Universidad de Buenos Aires (UBA)

ARGENTINA

E-mail: tomascifone@hotmail.com

Recibido : 24-06-2021

Aceptado: 01-08-2021 